Mass-stripping analysis of an interstellar cloud by a supernova shock

J. F. Hansen, H. F. Robey, A. R. Miles, R. I. Klein, C. F. McKee

May 7, 2006

HEDLA 2006

Houston, TX, United States

March 11, 2006 through March 14, 2006 
This document was prepared as an account of work sponsored by an agency of the United States Government. Neither the United States Government nor the University of California nor any of their employees, makes any warranty, express or implied, or assumes any legal liability or responsibility for the accuracy, completeness, or usefulness of any information, apparatus, product, or process disclosed, or represents that its use would not infringe privately owned rights. Reference herein to any specific commercial product, process, or service by trade name, trademark, manufacturer, or otherwise, does not necessarily constitute or imply its endorsement, recommendation, or favoring by the United States Government or the University of California. The views and opinions of authors expressed herein do not necessarily state or reflect those of the United States Government or the University of California, and shall not be used for advertising or product endorsement purposes. 


\title{
Mass-stripping analysis of an interstellar cloud by a supernova shock
}

\author{
J. F. Hansen, H. F. Robey, and A. R. Miles \\ Lawrence Livermore National Laboratory, Livermore CA 94550 \\ R. I. Klein \\ Lawrence Livermore National Laboratory, Livermore CA 94550 and \\ Department of Astronomy, University of California, Berkeley, CA 94720 \\ C. F. McKee \\ Departments of Physics and Astronomy, \\ University of California, Berkeley, CA 94720
}

\begin{abstract}
The interaction of supernova shocks and interstellar clouds is an important astrophysical phenomenon since it can result in stellar and planetary formation. Our experiments attempt to simulate this mass-loading as it occurs when a shock passes through interstellar clouds. We drive a strong shock using the Omega laser $(\sim 5 \mathrm{~kJ})$ into a foam-filled cylinder with an embedded $\mathrm{Al}$ sphere (diameter $D=120 \mu \mathrm{m}$ ) simulating an interstellar cloud. The density ratio between $\mathrm{Al}$ and foam is $\sim 9$. We have previously reported on the interaction between shock and cloud, the ensuing Kelvin-Helmholtz and Widnall instabilities, and the rapid stripping of all mass from the cloud. We now present a theory that explains the rapid mass-stripping. The theory combines (1) the integral momentum equations for a viscous boundary layer, (2) the equations for a potential flow past a sphere, (3) Spalding's law of the wall for turbulent boundary layers, and (4) the skin friction coefficient for a turbulent boundary layer on a flat plate. The theory gives as its final result the mass stripped from a sphere in a turbulent high Reynolds number flow, and it agrees very well with our experimental observations.
\end{abstract}




\section{INTRODUCTION}

We report here the results from experiments designed to study the interaction between a supernova shock and interstellar accumulations of matter (ISM) or "molecular clouds." The experiments were carried out at the Omega laser at the Laboratory of Laser Energetics in Rochester, New York [1]. We observe rapid stripping of all mass from a cloud, and we present a model based on turbulent boundary layers where the time scale of the mass-stripping agrees with the experiment. This may be important for understanding mass-loading in the astrophysical case, and for comparison to numerical simulations of the interaction between shocks and interstellar clouds which have shown that turbulence can generate structure which acts as seeds for star formation [2]. We note that aided by the forerunner of this experiment $[3,4]$, shock-cloud interaction evolved to a late time has recently been observed in supernova remnant Puppis A [5].

\section{EXPERIMENTAL SETUP AND DIAGNOSTICS}

The strong shock of a supernova explosion is simulated at experiments at Omega in the following manner: a small Be shock tube $(2.25 \mathrm{~mm}$ long; $0.8 \mathrm{~mm}$ inner diameter; $1.1 \mathrm{~mm}$ outer diameter) is filled with a low density $\left(300 \mathrm{mg} / \mathrm{cm}^{3}\right)$ carbonized resorcinol formaldehyde foam ( $\mathrm{CRF}$ ). The CRF at one end of the shock tube is then ablated by laser beams, causing the ejection of ablated material in one direction to launch a planar shock in the opposite direction. Good planarity of the shock is ensured by using multiple (nine), superimposed beams, each with a super-gaussian beam profile created by a phase plate in the focusing optics; the super-gaussian is of order eight and has a flat top matching the diameter of the shock tube. Each laser beam has an energy of $\sim 500 \mathrm{~J}$ with a pulse duration of $1.0 \mathrm{~ns}$.

The scaled ISM cloud is simulated by an $\mathrm{Al}$ sphere (radius $R_{0}=60 \mu \mathrm{m}$ ) embedded in the CRF a short distance into the shock tube (on the shock tube axis $500 \mu \mathrm{m}$ from the ablated CRF surface). The density ratio between the $\mathrm{Al}$ (density $2.7 \mathrm{~g} / \mathrm{cm}^{3}$ ) and the surrounding $\mathrm{CRF}$ is chosen to match the density ratio for an actual ISM cloud and other experimental parameters are also scaled to preserve the physics regime of the astrophysical case by following the guidelines set out by Ryutov [6]. Physical quantities in the CRF (without an embedded Al sphere) can be accurately calculated using the 1D radiative hydrodynamic code HYADES [7]. 


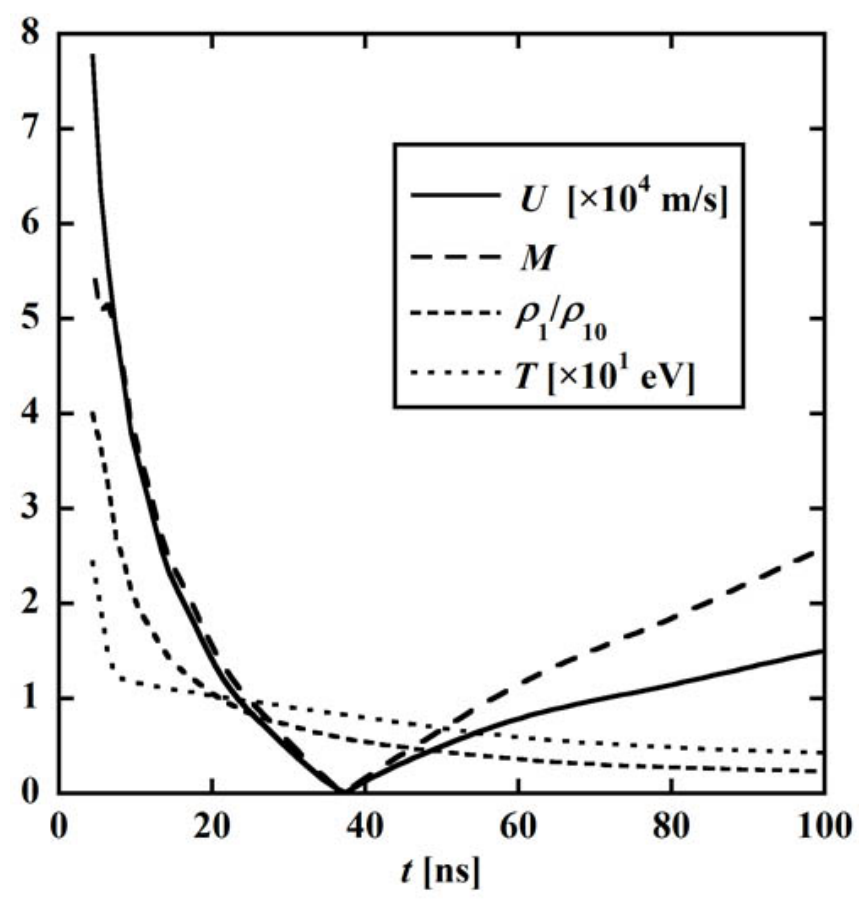

FIG. 1: HYADES calculation of the free-stream velocity $U_{\infty}=U_{\infty}(t)$, the Mach number $M$, the compression $\rho_{1}(t) / \rho_{10}$ (where the initial density $\rho_{10}=300 \mathrm{mg} / \mathrm{cm}^{3}$ ), and the temperature $T=T(t)$.

We have used this code to calculate the free-stream velocity $U_{\infty}(t)$, the temperature $T(t)$, and the density $\rho_{1}(t)$ for the CRF and these quantities are plotted in Fig. 1. (These values were used in our Euler scaling estimates above.) The simulation used an in-line quotidian equation of state (QEOS) model with a bulk modulus of $3 \times 10^{9} \mathrm{~Pa}$ and 112 zones to represent the $2.25 \mathrm{~mm}$ long CRF, with the first 46 zones feathered for ablation with a zone-to-zone scaling ratio of 1.15 , the final 46 zones feathered for shock release with a scaling ratio of 0.87 .

The cloud is imaged using a gated x-ray framing camera [8]. $\mathrm{X}$ rays for the image are generated by a second set of time-delayed laser beams (backlighter beams) pointed at a metal foil, typically $\mathrm{Ti}$, located on the opposite side of the shock tube from the camera. He- $\alpha$ radiation from the $\mathrm{Ti}$ (at $4.7 \mathrm{keV}$ ) moves through the shock tube and is imaged by either a $10 \mu \mathrm{m}$ pinhole located at the front end of the camera ("area radiography") or by a $20 \mu \mathrm{m}$ pinhole next to the Ti foil ("point projection radiography"). The point projection radiography technique results in a higher photon flux through the pinhole, and thus a better 
signal-to-noise ratio. The imaging element of the camera is either a microchannel plate (MCP) + film or MCP + charge coupled device (CCD), and in both cases has a size of $\sim 35 \mathrm{~mm}$. The exposure of the MCP was kept in the linear regime [9]. The distance from shock tube to $\mathrm{Ti}$ foil is $4.0 \mathrm{~mm}$ for area radiography, $6.5 \mathrm{~mm}$ for point projection radiography. The time-delay for the backlighter beams is chosen to obtain an image at a desired time $t$ after the initial, ablative laser pulse has started the shock in the shock tube. The camera MCP is triggered to coincide with the backlighter beams. The MCP pulse length was set to $500 \mathrm{ps}$ in a trade-off between maximizing x-ray exposure on the MCP while minimizing motion blurring (e.g., when the plasma moves $20 \mathrm{~km} / \mathrm{s}$ the motion blurring is $10 \mu \mathrm{m}$, comparable to the pinhole diameter). The experiment is repeated with different time-delays to generate an image sequence.

\section{RESULTS}

Results from the experiment can be seen in Fig. 2. As the shock runs over the cloud ( $t=$ $5 \mathrm{~ns}$ ), its speed inside the cloud is greatly reduced, leading to a Kelvin-Helmholtz instability and its characteristic roll-up ( $t=12 \mathrm{~ns})$. Soon thereafter, a Widnall-type instability [10] occurs, creating a low mode number azimuthal perturbation of order five when viewed from a point on the extended shock tube axis [4]. Here we see the Widnall instability as four "fingers" at the trailing edge of the cloud at $t=30 \mathrm{~ns}$, indicating a mode number of four to eight (depending on if each finger is or is not overlapping another finger along the line of sight). Material is constantly being stripped away from the $\mathrm{Al}$ plasma cloud and is visible in the images as a cone of diffuse material behind the cloud ( $t \geq 19 \mathrm{~ns}$ ). By $t=40 \mathrm{~ns}$ this cone extends outside our diagnostic field of view. By $t=60 \mathrm{~ns}$ so much material has been stripped away that the remaining cloud is quite diffuse (we are showing the $60 \mathrm{~ns}$ image at a higher contrast than the earlier point projection radiography images in Fig. 2). By $t=100 \mathrm{~ns}$ the cloud has been completely stripped away and can no longer be identified in the point projection radiography images. We also obtained an image at $80 \mathrm{~ns}$ in which the cloud is completely gone, but this target unfortunately had its Au spatial reference grid mounted outside the view of the x-ray framing camera and was therefore not included in Fig. 2.

Because the point projection radiography technique illuminates the MCP in a very uniform 


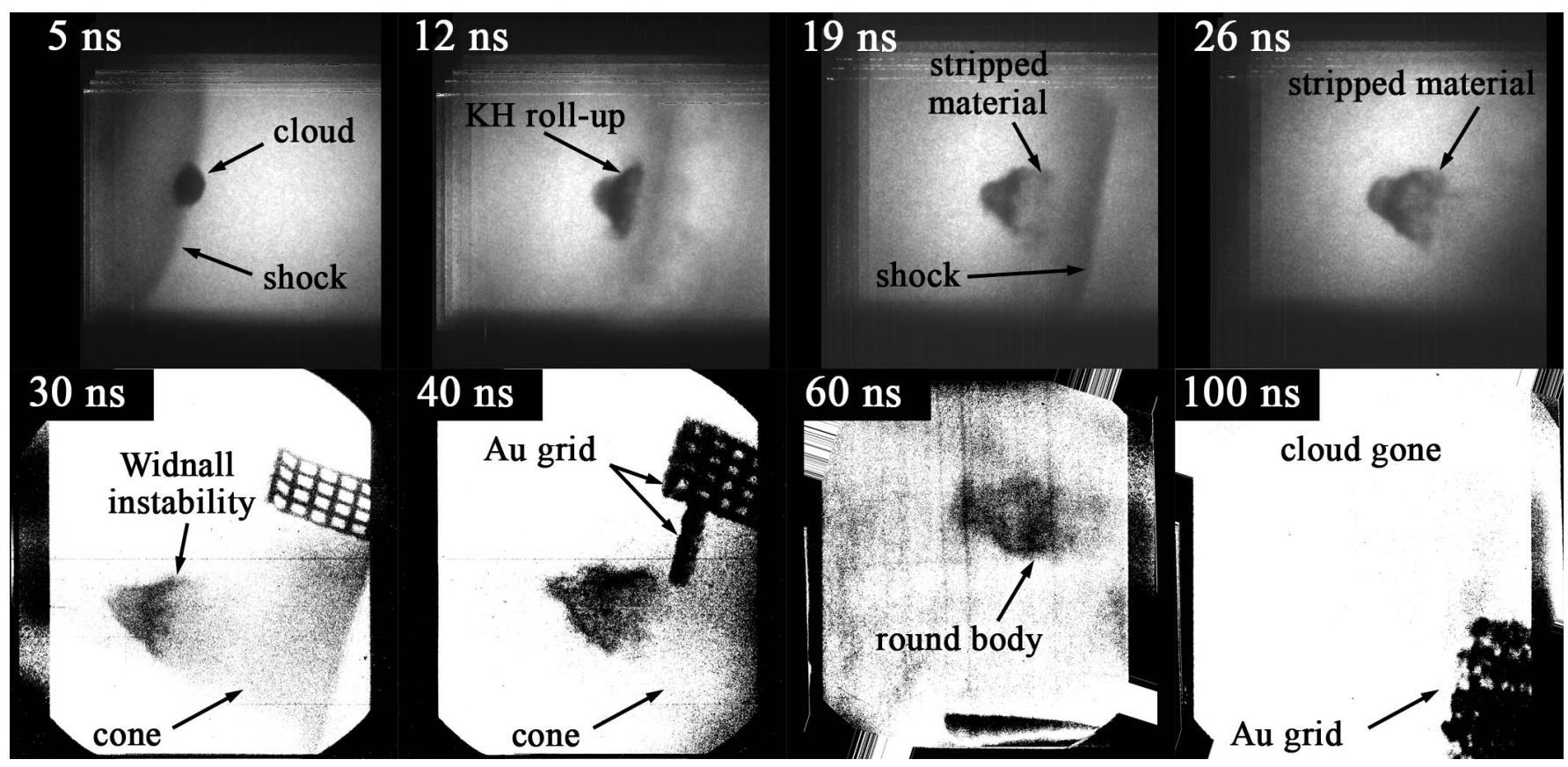

FIG. 2: A time sequence of images showing how the cloud evolves after the passage of a shock. In each image, the direction of motion of the shock is approximately from left to right, and is perpedicular to the imaged shock at time $t=19 \mathrm{~ns}$, and the imaged Au grids at times $t=30 \mathrm{~ns}$ and $40 \mathrm{~ns}$. In the first image, at $t=5 \mathrm{~ns}$, the shock is intersecting the cloud and the left hand side of the cloud is compressed by a factor of 4, the strong shock limit for a polytropic gas with an adiabatic index $\gamma=5 / 3$. The cloud undergoes a classical Kelvin-Helmholtz roll-up, as seen at $t=12 \mathrm{~ns}$ and later. Cloud material is stripped away from the cloud. Stripped material is clearly evident trailing the cloud at $t \geq 19 \mathrm{~ns}$ and is shaped as a cone that extends all the way to the shock (or extends outside the field of view at $t=40 \mathrm{~ns}$ ). A rarefaction changes the direction of the surrounding flow at approximately $t=40 \mathrm{~ns}$, and by $t=60 \mathrm{~ns}$ the reverse flow has caused the right hand side of the cloud to become fairly round. By $t=100 \mathrm{~ns}$ the cloud has disappeared. The first four images were obtained with area backlighters, the last four with point projection radiography.

fashion, we can use the point projection radiography images to estimate the cloud mass using the formula $I=I_{0} \exp (-m / \mu A)+I_{b}$ where $I$ is the measured pixel intensity, $I_{0}$ is the $\mathrm{x}$ ray source intensity (i.e., the intensity we would have expected to measure had there not been mass attenuation), $m$ is the integrated line of sight mass, $\mu$ is the x-ray attenuation coefficient in units of mass per area, $A$ is the pixel area in the image, and $I_{b}$ is background 
intensity from all sources of non-directional exposure, such as film fogging, non-directional $\mathrm{x}$ rays, energetic particles, etc. We estimate the cloud mass to be $0.67 \pm 0.11 \mu \mathrm{g}$ at $t=30 \mathrm{~ns}$ and $0.54 \pm 0.11 \mu \mathrm{g}$ at $t=40 \mathrm{~ns}$ (it is coincidental that the error is $0.11 \mu \mathrm{g}$ in both of these images - the error is normally different from image to image). This can be compared to the original sphere mass of $2.44 \mu \mathrm{g}$.

\section{ANALYSIS}

We present in this section a new mathematical model that describes mass stripping from a cloud under turbulent, high Reynolds number conditions. We compare this model to our experimental data and to an existing model $[11,12]$ for laminar mass stripping. Our model combines four separate concepts of fluid mechanics: (1) the integral momentum equations for a viscous boundary layer, (2) the equations for a potential flow past a sphere, (3) Spalding's law of the wall for turbulent boundary layers [13], and (4) the skin friction coefficient for a turbulent boundary layer on a flat plate.

We begin with the integral momentum equations for a stationary, viscous boundary layer:

$$
\begin{gathered}
\frac{\partial}{\partial x} \int_{0}^{\delta_{1}} u_{1}\left(U-u_{1}\right) \mathrm{d} y_{1}+\frac{\mathrm{d} U}{\mathrm{~d} x} \int_{0}^{\delta_{1}}\left(U-u_{1}\right) \mathrm{d} y_{1}+\frac{1}{r} \frac{\mathrm{d} r}{\mathrm{~d} x} \int_{0}^{\delta_{1}} u_{1}\left(U-u_{1}\right) \mathrm{d} y_{1}=\left.\nu_{1} \frac{\partial u_{1}}{\partial y_{1}}\right|_{y_{1}=0} \\
\frac{\partial}{\partial x} \int_{0}^{\delta_{2}} u_{2}^{2} \mathrm{~d} y_{2}+\frac{1}{r} \frac{\mathrm{d} r}{\mathrm{~d} x} \int_{0}^{\delta_{2}} u_{2}^{2} \mathrm{~d} y_{2}+\frac{1}{\rho_{2}} \frac{\mathrm{d} p}{\mathrm{~d} x} \delta_{2}=-\left.\nu_{2} \frac{\partial u_{2}}{\partial y_{2}}\right|_{y_{2}=0} \\
\left.\rho_{1} \nu_{1} \frac{\partial u_{1}}{\partial y_{1}}\right|_{y_{1}=0}=-\left.\rho_{2} \nu_{2} \frac{\partial u_{2}}{\partial y_{2}}\right|_{y_{2}=0}
\end{gathered}
$$

where $x$ is a coordinate along the surface of the cloud (we will approximate the cloud with a sphere at all times so that $x=0$ at the flow stagnation point and $x=\frac{\pi}{2} R$ at the equator), $y$ is a coordinate perpendicular to the cloud surface, $r$ is the distance from the cloud surface to the cloud axis of symmetry, $U=U(x)$ is the free stream flow velocity behind the shock, $u=u(x, y)$ is the flow velocity inside the boundary layer, $\nu$ is the kinematic viscosity, $\rho$ is the density, $p=p(x)$ is the pressure, and $\delta=\delta(x)$ is the thickness of the boundary layer. The flow properties $U, u, \nu, \rho, p$, and $\delta$ are also functions of time $t$. Subscript 2 denotes 


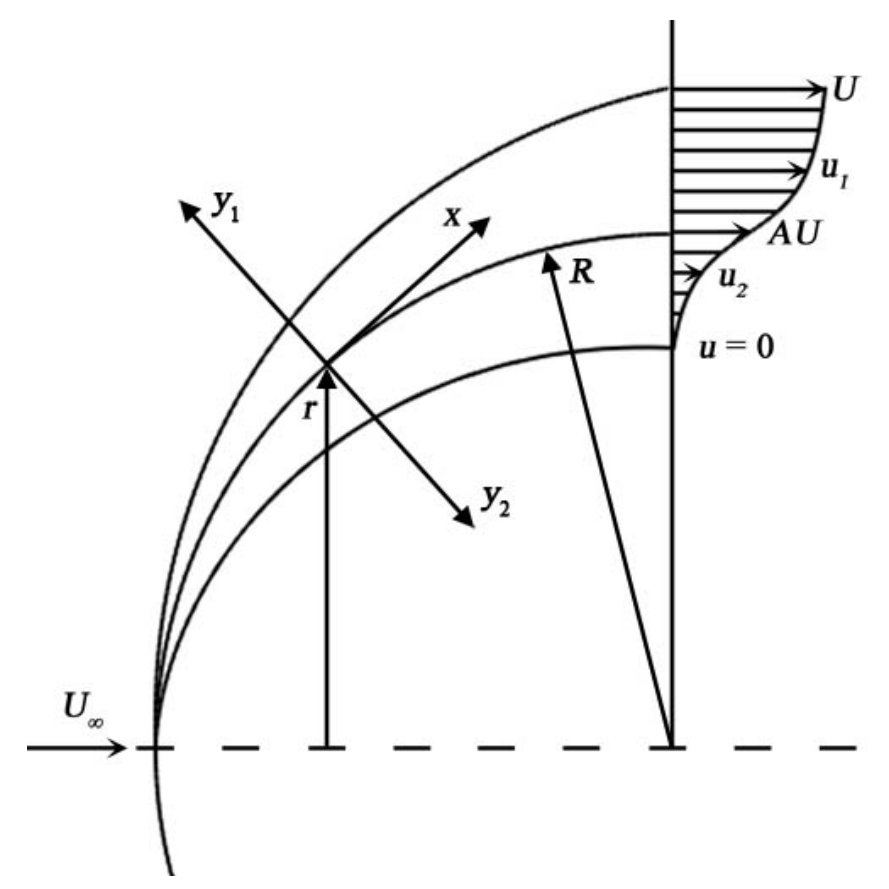

FIG. 3: The flow geometry around the cloud is modelled with the potential flow around a sphere with the boundary layer flow calculated using a local cartesian coordinate system where the coordinate $x$ is along the flow (i.e., along the surface of the sphere) and $y$ is the distance into the boundary layer from the sphere surface. The velocity in the boundary layer is $u_{1}$ outside the sphere radius $R$ and $u_{2}$ inside the sphere radius. At the outside edge of the boundary layer $y_{1}=\delta_{1}$ the velocity $u_{1}=U$, i.e., matches the potential flow velocity. At the sphere radius $u_{1}=u_{2}=A U$, where $A$ is a constant. At the inside edge of the boundary layer $y_{2}=\delta_{2}$ the velocity $u_{2}=0$. The cylindrical coordinate $r$ is the distance from the axis of symmetry to the sphere surface.

plasma in the cloud and subscript 1 the surrounding flow, e.g., $\delta_{2}$ is the boundary layer thickness inside the cloud. The geometry is sketched out in Fig. 3.

We will assume that the free stream velocity around the cloud follows the potential flow of a sphere,

$$
U(x)=\frac{3}{2} U_{\infty} \sin \left(\frac{x}{R}\right),
$$

where $U_{\infty}$ is the flow velocity far from the sphere. This has the advantage that $\mathrm{d} U / \mathrm{d} x=0$ at the equator $x=\pi R / 2$, which will simplify the algebra substantially. Also, at the equator $\mathrm{d} p / \mathrm{d} x=-\rho_{g} U \mathrm{~d} U / \mathrm{d} x=0$ and trivially $\mathrm{d} r / \mathrm{d} x=0$

To express the boundary layer velocity $u$ as a function of the distance $y$ from the sphere 
surface, we will use Spalding's law of the wall for turbulent boundary layers [13]:

$$
y^{+}=u^{+}+e^{-\kappa B}\left(e^{\kappa u^{+}}-1-\kappa u^{+}-\frac{\left(\kappa u^{+}\right)^{2}}{2}-\frac{\left(\kappa u^{+}\right)^{3}}{6}\right)
$$

where the dimensionless coordinate $y^{+} \equiv y v^{*} / \nu$ and the dimensionless velocity $u^{+} \equiv u / v^{*}$, or in the appropriate coordinate frame of reference $u_{1}=v_{1}^{*} u_{1}^{+}+A U$ and $u_{2}=A U-v_{2}^{*} u_{2}^{+}$, where $A$ is a constant such that $A U$ is the flow velocity on the sphere surface. The wallfriction velocity $v^{*}$ is defined through $v^{*^{2}}=\left.\nu(\mathrm{d} u / \mathrm{d} y)\right|_{y=0}$ and Coles [14] gives the coefficients $\kappa=0.41$ and $B=5.0$.

This is as far as we can go without saying something about the wall-friction velocity $v^{*}$, or equivalently the skin-friction coefficient $C_{f}$, as the two are related through

$$
v^{*^{2}}=\frac{1}{2} C_{f}(x) U^{2}(x) .
$$

For calculations of skin-friction drag, many renowned researchers, beginning with Dryden [15] and Millikan [16], have used velocity distributions for a flat plate in non-flat geometries and found that the results do not differ seriously from measured values [17]. We will do the same and use the skin friction coefficient for a turbulent boundary layer on a flat plate [18]:

$$
C_{f}(x) \approx 0.0592 \operatorname{Re}_{x}^{-1 / 5}
$$

where the Reynold's number $\operatorname{Re}_{x}=U x / \nu$, but with a modification; if we use Eq. 7 as is, the problem is overdetermined. We replace the coefficient 0.0592 with a coefficient that will be determined by our system of equations. We set:

$$
C_{f}(x)=\frac{2}{\alpha^{2}}(U x / \nu)^{-1 / 5}
$$

We can now rewrite Eq. 1 as

$$
\begin{aligned}
1200 \kappa^{2} e^{\kappa B} K_{1}^{9}(1-A) \alpha_{1}^{-1}=120 e^{\xi}\left[(1+2 A) \xi^{2}-(3+2 A) \xi+4\right]-3(3+10 A) \xi^{5}+ \\
-20(1+A) \xi^{4}+20\left(\kappa^{-1} e^{\kappa B}-1\right)(1+2 A) \xi^{3}-120(1-2 A) \xi-480
\end{aligned}
$$

where

$$
\xi=\kappa K_{1}(1-A) \alpha_{1}
$$




$$
K_{1}=\left(\frac{3 \pi U_{\infty} R}{4 \nu_{1}}\right)^{1 / 10}
$$

We expect $A$ to be a fairly small quantity (it will certainly be smaller than unity) so one might be tempted to linearize Eq. 9 w.r.t. $A$, but this only simplifies terms where $A$ (or $\xi$ ) does not appear in the exponents and does not lead to an analytical solution for $A$ (as it does in the laminar model). Consequently some form of simple numerical scheme must be employed to calculate $A$, and we have therefore chosen to not linearize Eq. 9 w.r.t. $A$, but to keep the exact form.

Similarly in the Al cloud defining

$$
K_{2}=\left(\frac{3 \pi U_{\infty} R}{4 \nu_{2}}\right)^{1 / 10}
$$

allows us to rewrite Eq. 2 as

$$
600 \kappa^{2} e^{\kappa B} K_{2}^{9} \alpha_{2}^{-1}=120 e^{\eta}(\eta-2)-3 \eta^{5}-10 \eta^{4}+20\left(\frac{1}{\kappa} e^{\kappa B}-1\right) \eta^{3}+120 \eta+240
$$

where

$$
\eta=\kappa K_{2} A \alpha_{2}
$$

Next relate $\alpha_{1}$ to $\alpha_{2}$ by rewriting Eq. 3 as

$$
\alpha_{1}=K_{3} \alpha_{2}
$$

where

$$
K_{3}=\left(\frac{\rho_{1}}{\rho_{2}}\right)^{1 / 2}\left(\frac{\nu_{1}}{\nu_{2}}\right)^{1 / 10} .
$$

Eliminate $\alpha_{1}$ by substituting Eq. 15 in Eqs. 9-10, leaving us with two equations, Eqs. 9 and 13, for two unknown coefficients $A$ and $\alpha_{2}$. This equation pair can easily be solved numerically, e.g., Eq. 9 can be solved for $A$ by simple iteration as it converges nicely, and a simple regula falsi (secant) method can be used for Eq. 13, but other numerical schemes will work, too, and we used a globally convergent Newton's method. With $A$ and $\alpha_{2}$ at hand, one easily calculates the mass stripped from the cloud by integrating the cloud material flowing through the boundary layer at the equator [12]:

$$
\frac{\mathrm{d} m}{\mathrm{~d} t}=2 \pi R \rho_{2} \int_{0}^{\delta_{2}} u_{2} \mathrm{~d} y_{2}=2 \pi R \rho_{2} \nu_{2} \psi(\eta)
$$


where we have defined a mass-strip coefficient

$$
\psi(\eta)=\frac{1}{2 \kappa^{2}} \eta^{2}+\frac{1}{\kappa} e^{-\kappa B}\left(1-\eta-\frac{1}{2} \eta^{2}-\frac{1}{6} \eta^{3}-\frac{1}{24} \eta^{4}+e^{\eta}\right)
$$

It should be noted that $\mathrm{d} m / \mathrm{d} t$ is not proportional to $R, \rho_{2}$, or $\nu_{2}$ because $\eta=$ $\eta\left(R, U_{\infty}, \rho_{1}, \rho_{2}, \nu_{1}, \nu_{2}\right)$ from the numerical solution above.

Using the specific physical quantities for our experiment, we can now calculate the mass stripped as a function of time and see how the calculation compares to our experimental data. For the cloud radius $R(t)$ we use measured values from the experiment images, and interpolate to other times. For values of the free stream flow velocity $U_{\infty}(t)$, the density $\rho_{1}(t)$, and the temperature $T(t)$, we use values from HYADES. The density $\rho_{2}(t)$ is obtained by applying the same compression as for $\rho_{1}(t)$. All of these values are in full agreement with values from CALE. Additionally, the peak compressions are independently verified from the experiment at $t=5 \mathrm{~ns}$ where the left side of the sphere is compressed to an ellipsoid shape with minor radius $\sim 30 \mu \mathrm{m}$, corresponding to a compression of $\sim 4$ (which is the strong shock limit for a polytropic gas with adiabatic index $\gamma=5 / 3$ ).

With our given physical quantities, the coefficients $K_{1} \approx 5, K_{2} \approx 3$, and $K_{3} \approx \frac{1}{4}$ at all times. From solving Eqs. 9, 13 and 15 we calculate the coefficients $A \approx 1 / 5, \alpha_{1} \approx 6$, and $\alpha_{2} \approx 27$ at all times, and we find that the compound quantity $\eta$ varies between $4 \lesssim \eta \lesssim 8$ (except very briefly when the rarefaction changes the direction of the flow) so that the mass-strip coefficient $\psi$ is in the range $4 \times 10^{2} \lesssim \psi \lesssim 4 \times 10^{3}$. The mass of the cloud as a function of time is plotted in Fig. 4 and reaches $m=0$ (fully stripped) by $t \approx 90 \mathrm{~ns}$. This agrees well with the experiment where the cloud can no longer be observed by $80 \mathrm{~ns}-100 \mathrm{~ns}$. By comparison, the equivalent mass-strip coefficient $\psi=\left(2 \pi R \rho_{l} \nu_{l}\right)^{-1} \mathrm{~d} m / \mathrm{d} t$ in the laminar model is $\lesssim 4 \times 10^{2}$ for all times of interest in the experiment, which is too low to achieve the cloud being completely stripped by $t \approx 80 \mathrm{~ns}$; if the mass-stripping was done by laminar flow and continued under the same conditions past $t=80 \mathrm{~ns}$ (ignoring experimental limitations) the laminar mass-stripping time would be $\sim 1 \mu \mathrm{s}$. As a final note, to illustrate the nonlinearity between $\mathrm{d} m / \mathrm{d} t$ and the various physical quantities one can arbitrarily double, say, the value of the viscosity $\nu_{2}$ and see that this leads to only a $12 \%$ increase in $\mathrm{d} m / \mathrm{d} t$. 


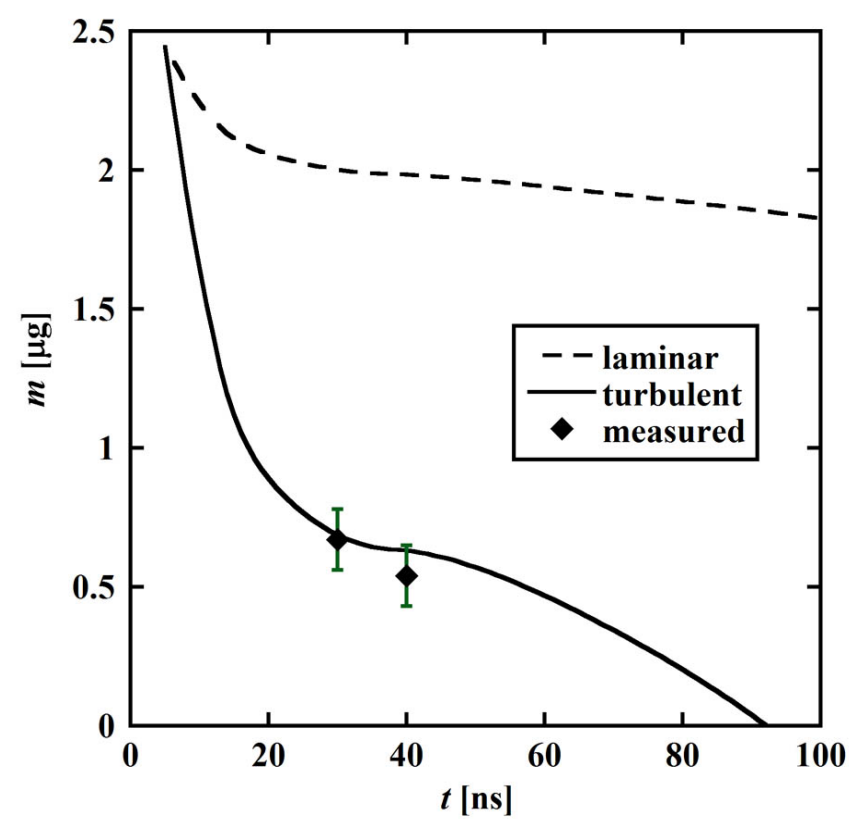

FIG. 4: Cloud mass remaining as a function of time calculated using a laminar model [11, 12] (dashed line) and the turbulent model presented in this manuscript (solid line), compared to experimentally measured values of the cloud mass (two squares). The turbulent model agrees with the measured values and also predicts that the cloud is completely stripped by $\sim 90 \mathrm{ns,} \mathrm{which}$ compares well with the experimental observation of the cloud being stripped by $80 \mathrm{~ns}-100 \mathrm{~ns}$. In the laminar model (assuming unchanged condition from $80 \mathrm{~ns}$ ) the cloud is not stripped until $\sim 1 \mu \mathrm{s}$.

\section{SUMMARY}

We observe the rapid stripping of all mass from a simulated interstellar cloud in a laser experiment. We present a model that agrees very well with our experimental observations. The model combines (1) the integral momentum equations for a viscous boundary layer, (2) the equations for a potential flow past a sphere, (3) Spalding's law of the wall for turbulent boundary layers, and (4) the skin friction coefficient for a turbulent boundary layer on a flat plate. By comparison, a laminar model overestimates the stripping time by an order of magnitude. This suggests that mass-stripping in the experiment must be of a turbulent nature, and with its even higher Reynolds numbers, this must hold also in the astrophysical case.

This work was performed under the auspices of the U. S. Department of Energy by 
the University of California, Lawrence Livermore National Laboratory under Contract No. W-7405-Eng-48.

[1] T. R. Boehly, D. L. Brown, R. S. Craxton, R. L. Keck, J. P. Knauer, J. H. Kelly, T. J. Kessler, S. A. Kumpan, S. J. Loucks, S. A. Letzring, F. J. Marshall, R. L. McCrory, S. F. B. Morse, W. Seka, J. M. Soures, and C. P. Verdon, Optics Communications, 133, 495 (1997).

[2] P. C. Clark and I. A. Bonnell, Mon. Not. R. Astron. Soc., 361, 2 (2005).

[3] R. I. Klein, K. S. Budil, T. S. Perry, and D. R. Bach, Astrophys. J. Suppl. S., 127, 379 (2000).

[4] H. F. Robey, T. S. Perry, R. I. Klein, J. O. Kane, J. A. Greenough, and T. R. Boehly, Phys. Rev. Lett., 89, 085001 (2002).

[5] U. Hwang, K. A. Flanagan and R. Petre, Astrophys. J., 635, 355 (2005).

[6] D. Ryutov, R. P. Drake, J. Kane, E. Liang, B. A. Remington and W. M. Wood-Vasey, Astrophys. J., 518, 821 (1999).

[7] J. T. Larsen and S. M. Lane, J. Quant. Spectrosc. Radiat. Transfer, 51, 179 (1994).

[8] K. S. Budil, T. S. Perry, P. M. Bell, J. D. Hares, P. L. Miller, T. A. Peyser, R. Wallace, H. Louis and D. E. Smith, Rev. Sci. Inst., 67, 485 (1996).

[9] O. L. Landen, P. M. Bell, J. A. Oertel, J. J. Satariano, and D. K. Bradley, UCRL-JC-112384 Gain Uniformity, Linearity, Saturation and Depletion in Gated Microchannel-Plate X-Ray Framing Cameras (Lawrence Livermore National Laboratory, Livermore, 1994). Copies may be obtained from the National Technical Information Service, Springfield, VA 22161.

[10] S. E. Widnall, D. B. Bliss and C. Tsai, J. Fluid Mech., 66, 35 (1974).

[11] G. I. Taylor, "The Shape and Acceleration of a Drop in a High Speed Air Stream." In the Scientific Papers of G. I. Taylor, ed. G. K. Batchelor, University Press, Cambridge (1963).

[12] A. A. Ranger and J. A. Nicholls, AIAA Journal 7, 285 (1969).

[13] D. B. Spalding, J. Appl. Mech., 28, 455 (1961).

[14] E. Coles, In Fifty Years of Boundary Layer Research, ed. W. Tollmien and W. Görtler, Vieweg, Brunswick (1955).

[15] H. L. Dryden and A. M. Kuethe, N.A.C.A. Report, 342, 12 (1930).

[16] R. A. Millikan, Trans. Amer. Soc. Mech. Engineers, 54, 29 (1932).

[17] "Modern Developments in Fluid Dynamics," ed. S. Goldstein, Dover, New York (1965). 
[18] F. M. White, "Viscous Fluid Flow," McGraw-Hill, New York (1974). 